\title{
XX. Ueber die Umwandlung des Quarzes in Speckstein.
}

\author{
Von \\ E. Woinschenk in Munchen.
}

(Hierzu Tafel VII.)

Literatur.

Breith a p t, Uiber die Aechlheit der Kristalle. 1815.

$S$ teffens, Vollständiges Lehrbuch der Oryktognosie.

Haüy, Comparaison des résultats de la crystallographie et de l'analyse chimique.

B l um, Die Pseudomorphosen des Mineralreichs nebst 4 Nachträgen, $1843 \mathrm{ff}$.

$\mathrm{N}$ a uck, "Das Specksteinlager zu Göpfersgrün “. In PoggendorfI's Annalen 1848, 75, $129 \mathrm{ff}$.

B is chof, Chemische und physikalische Geologie. 1865.

Geinitz, "Ueber Pseudomorphosen «. Neues Jahrbuch für Mineralogie etc. 1876, 491. Sandberger, Sitzungsberichte der Münchner Akademie. 1872, 12.

G ü m bel, Geognostische Beschreibung des Fichtelgebirges. 1879, 172. (Nebst Karten.)

Im südöstlichen Theile des Fichtelgebirges findet sich ein ausgedehntes Specksteinlager, das schon fruhe das Interesse der Mineralogen erregt hat und zwar namentlich durch die Mannigfaltigkeit der Formen, in denen der Speckstein hier pseudomorph auftritt. Unter diesen zeicbnen sich besonders die Pseudomorphosen nach Quarz und Bitterspath durch die Häufigkeit ihres Vorkommens und die Schönheit ihrer Individuen aus.

Man suchte dieselben zuerst als Krystalle von Speckstein aufzufassen, doch kam man sehr bald von dieser Ansicht zuruck und stellte die Theorie einer Pseudomorphosirung aus Quarz respective Bitterspath auf.

Breithaupt sagt hiertber: "So wenig nun jene Specksteinkrystalle für ächte genommen werden dürfen, ebensowenig haben sie die Charaktere der pseudomorphischen “*), und weiterhin bemerkt er: "Lange konnte ich

*) Breith a upt fasst den Begriff „Pseudomorphosen « enger, als wir es heutzutage zu thun pflegen. Unter "Pseudomorphosen * versteht er ausschliesslich unsere heutigen Ausfüllungspseudomorphosen.

Gr ot h, Zeitschrift f. Krystallogr. XIV. 
nicht an eine chemische Umwandlung des Quarzes in Speckstein glauhen, denn der gegenwärtige Zustand der Chemie kann uns eine solche Melamorphose nicht erklären."

Auch Ste ffens ist der Ansicht, dass hier eine Umwandlung vorliegt: "Also entstehen alle krystallisirten Formen des Speckstoins, ja höchst wahrschéinlich aller Speckstein durch Metamorphose, eine Umbildung derjenigen analog, durch welche mittels der Fäulniss thierischer Stoffe Wallrath und talgähnliche Substanzen gebildet werden. «

Diesen Theorien tritt $\mathrm{Ha}$ a y entgegen; er findet »einen doppelten Belag für die Meinung, dass hier der Speckstein Gestalten zeige, welche in Eindrücken gebildet sind; die Krystalle, von denen sich letztere herschreiben, existirten früher und haben der neuen Substanz ihren Platz eingeräumt. « Er führt auch die höchst abenteuerliche Hypothese von B roch ant auf, welcher glaubt, die Krystalle seien ein inniges Gemenge von Quarz und Speckstein, ähnlich wie der Kalkspath von Fontainebleau.

B lu m kommt zu dem Schlusse, die Bittererde des Dolomits habe sich mit der Kieselsäure des Quarzes zu Speckstein verbunden.

Hiergegen fuhrt $\mathrm{Nau}$ ck in seiner sehr umfassenden Arbeit, in welcher er namentlich die geognostischen Verhältnisse sehr eingehend und treffend schildert, an, „dass der Magnesiagehalt des Bitterspathes nicht hingereicht haben wurde, um sowohl diesen, als den Quarz ohne Volumenverlust in Speckstein umzuwandeln und noch obendrein alle $Z$ wischenräume mit Speckstein auszufüllen (. Er fuhrt des Weiteren aus: „Dieses Deficit deckt Blum durch die Annahme einer Magnesiaexhalation (!). Es seien wahrscheinlich bei der Bildung der Augitporphyre Magnesiadämpfe aufgestiegen, vielleicht zwischen den Sprünen des Granits yon Thiersheim $u$. s. w. Dieser gasförmigen Magnesia wird nun ausser der Dolomitisation des Urkalkes u. a. auch die Bildung des Specksteins zugeschrieben; abgesehen von der Räthselhaftigkeit des ganzen Vorganges, möchte doch, wenn er wirklich statuirt werden sollte, seine Anwendung auf den vorliegenden Fall manchen wichtigen Punkt unerklärt lassen, namentlich die vollkommene Erhaltung der Krystallform. Auch die Ausfullungsmasse zwischen den Krystallen, dieser Stein des Anstosses für alle bisherigen Erklärungsversuche, ist durchaus nicht genugend erklärt. « Und schliesslich kommt er zu dem Resultate: ..... es wird zur evidenten Gewissheit, dass der Speckstein von aussen her als Magnesiasilicat zugeführt wurde ", wobei er jedoch in seiner 'ganzen Arbeit durchgebends vou einer Umwandlung spricht.

Der gleichen Ansicht ist auch Bischof, er spricht sich dahin aus: „Wo, wie zu Göpfersgrün, so verschiedenartige Mineralien, wie Quarz, Dolomit und Bitterspath, in Speckstein umgewandelt sind, kann das Material des ursprünglichen Minerals keinen Einfluss gehabt haben. " 
All' diese Ansichten basiren auf der makroskopischen Betrachtung des Vorkommens von Göpfersgriun, sowie auf dem eingehenden Studium der geognostischen Verhältnisse. Mikroskopisch wurde es zum erstenmale von Geinitz untersucht; dieser fand jedoch "keine bedeulungsvollen Argumente, ob Umwandlungs- oder Verdrängungspseudomorphosen im Sinne B I u m's vorliegen «.

Sandberger geht von der Betrachtung der Pseudomorphosen von Olomutschan in Mähren aus, die er auf Grund ihrer theilweisen Löslichkeit in Kalilauge für "Kacholong nach Quarz « erklärt und sảgt im Zusammenhange damit: "Die Umwandlung von Quarz in amorphe Kieselsäure gab zum erstenmale Aufschluss über die Art, auf welche die so lange räthselhaften Pseudomorphosen von Speckstein nach Quarz sich gebildet haben. Die Aufnahme von Basen hat, bei vorheriger Umwandlung in amorphe Kieselsäure, durchaus keine Schwierigkeit. “

Gï m bel spricht sich nur ganz allgemein für eine Umwandlung von Quarz und Dolomit in Speckstein aus, indem er zugleich auf die Contactwirkungen des Granits hinweist.

Das Specksteinlager von Göpfersgrün befindet sich etwa $6 \mathrm{~km}$ ostnordöstlich von Wunsiedel im Fichtelgebirge an der Grenze von Granit und krystallinischen Schiefern, welche aus Glimmerschiefer und Phyllit, seltener aus Serpentin, Variolit und Chlorilschiefer bestehen, und bäufige Uebergänge unter einander zeigen. Es bildet einen Theil eines Kalkzugs, der ziemlich steil nach Suden einfallend den krystallinischen Schiefern in ostnordöstlicher Richtung eingelagert ist und bei Göpfersgrun, wo er sich dem eruptiven Centralgranitstock des Fichtelgebirges nähert, etwas nach Sưden gedrängt und steiler aufgerichtet erscheint. Dieser Zug lässt sich mit häufigen Unterbrechungen von Eulenlobe uber Göpfersgrïn bis Hohenberg verfolgen und bildet in der letzteren Partie die Grenze der krystallinischen Schiefer gegen Granit, Basalt und Augitporphyr.

Parallel damit streicht elwas sudlicher ein ähnliches Kalklager durch die krystallinischen Schiefer, das, gleichfalls vielfach unterbrochen, von Ebnath uber Pullenreuth, Redwitz, Arzberg bis Schirnding sich erstreckt. Beide Züge bestehen aus einem grobkörnigen, weissen bis graulich- oder gelblichweissen Kalkstein, der meist in dunnen Platten bricht und deshalb nicht zu Kunstwerken verwendet werden kann. Nicht selten ist er in Dolomit umgewandelt, mit dem er auch häufig wechsellagert.

Beide Zuge sind reich an Spatheisensteineinlagerungen, der an mehreren Stellen bergmännisch ausgebeutet wird und den Namen "Weisserz" fuhrt. In den oberen Teufen ist er meist in Brauneisenstein umgewandelt und zeigt reichliche Einlagerungen und Ueberzige von Ghalcedon. 
Sehr charakteristisch sind für die Kalke die Quarzbildungen, die, namentlich an der Grenze gegen Granit und Basalt, die Spalten erfullen und sehr häufig in Drusen neben Bitterspath auskrystallisirt sind. Auch der ganze Kalk selbst ist nicht selten mit kleingen Quarzkörnchen imprägnirt und erscheint dann etwas härter.

Besonders interessant ist in dieser Beziehung der Strehlerberg bei Redwitz, der dem südlichen Zuge angehörend, der Lage und dem Vorkommen nach dem Speckstein des nördlichen entspricht. Es bricht hier ein echter Dolomit, der aber in engster Beziehung zu dem Urkalk von Redwitz stebt, und in den Drusen und Spalten desselben findet sich neben Bitterspath schöner wasserheller Quarz auskrystallisirt; beide sind häufig verwachsen und zwar so, dass der Quarz Eindrücke von Rhomboëdern zeigt, oder aber ganz von denselben umhullt ist; eine gleichzeitige Bildung beider durfte darum kaum zweifelhaft erscheinen. Aehnliche Stücke finden sich, wenn auch selten, in der Nähe von Göpfersgrün.

Ausser diesen Bildungen umschliesst der Urkalk, namentlich da, wo er sich dem Granit und Basalt nähert, eine grosse Anzahl von Mineralien, z. B. Fluorit, Ghondrodit, Magnetkies, Schwefelkies (die beiden letzteren auch zu Brauneisen umgewandelt), Granat, Phlogopit, Grammatit, Graphit und viele andere.

Einige von diesen finden sich pseudomorphosirt im Specksteinlager selbst vor, so der Grammatit; die Pseudomorphose zeigt die faserig stenglige Textur des ursprünglichen Minerals und lässt deutlich erkennen, dass in seiner Umgebung der Dolomit grobkörniger geworden ist. Fruher sollen auch Pseudomorphosen nach Flụorit vorgekommen sein, doch gelang es mir nicht, etwas Derartiges aufzufinden, ebensowenig wie die von Blum erwähnten Pseudomorphosen nach Granat, Turmalin und Spinell aus dem Granit von Thiersheim; dagegen findet sich nicht selten der im Dolomit zu Bändern angeordnete körnige Graphit in der gleichen Anordnung im Speckstein wieder und weist deutlich auf die Entstehung des letzteren aus dem Dolomit hin.

Die Mehrzahl der oben aufgezählten Mineralien sind solche, wie sie auch an andern Orten den Contactwirkungen von Eruptivgesteinen zugeschrieben werden, und es dürfte ihr Vorkommen ebenso wie das des Egerans, der sich unweit von Göpfersgrün im Kalke findet, in unmiltelbarstem Zusammenhange mit den Eruptionen von Granit und Basalt stehen. Die beiden letzteren sind an der Grenze stark zersetzt und ausgelaugt, der Granit ist theilweise ganz in Speckstein umgewandelt, theils zu Kaolin verwittert; doch enthält er meist noch Reste des ursprunglichen Quarzes. Phyllit und Glimmerschiefer sind sebr weich und fettig geworden und bestehen aus Speckstein, der aber stets Thon beigemengt enthält. Die schiefrige Textur und die Farbe haben diese umgewandelten Gesteine beibehalten. 
Der Kalk wird, gegen den Granil zu, immer Magnesia-reicher, indem die Dolomitisirung theils abwechselnde Schichten, theils das ganze Lager ergreift. Sehr haufig sind dann auf Drusen und Spalten des Gesteins Bilterspathkrystalle, die in der Nähe des Specksteinlagers die ersten Alizeichen der Zersetzung darbieten. Die Krystalle sind weisslich trube geworden und sehen wie gebrannt aus; seltener zeigen sie eineu oberflächlichen Absatz von Eisen- oder Manganoxydhydrat. Der Dolomit selbst wird gelblich und fettig, und lässt mit Salzsäure behandelt einen unlöslichen Rückstand, der weiter zerlegt sich als Magnesiasilicat zu erkennen giebt; auch sieht man in demselben Schuppen und Bulzen von Speckstein.

Unter dem Mikroskope zeigen die Slücke im ersten Stadium der Umwandlung eine Trübung der Individuen durch feine Capillarkanalsysteme; daneben setzen sich auf den Spaltungsrichtungen doppel tbrechende Schuppchen ab (Taf. VII, Fig. I links). Diese häufen sich namențlich an den Punkten, wo viele derartige natürliche Kanäle zusammenstossen, immer mehr an und greifen in die noch unzerselzle Masse viber, bis endlich nur noch rhomboëdrische Skelette von trübem Dolomit in der schuppig filzigen Grundmasse eingebettet liegen (Fig. 1 rechts unlen). Derarlige Stucke finden sich in den liefsten, erst neuerdings durch die Gruben aufgeschlossenen Theilen des Lagers, und daneben zeigen sich auf den theils noch frischen theils schon angegriffenen Bitterspathkrystallen, die sich in den Hohlräumen gebildet baben, beerenförmige Absätze von reinem Speckstein, die sich leicht von den darunter befindlichen Krystallen ablösen lassen.

Der unmiltelbar über diesen Bildungen vorkommende Speckstein bricht in Knollen, seltener in Platten, die in einem wasserreichen, fettig-thonigen Mulm eingebeltel sind und ein Gewicht bis zu mehreren Centnern erreichen. Seine Farbe ist selten rein weiss; gelbliche bis grünliche Partien sind volherrschend, doch finden sich auch dunkelgrüne mit allerlei Flecken versehene Stücke, die sich von Augitporphyren oder ähnlichen Gesteinen abzuleiten scheinen. Häufig isı er von Dendriten durchzogen, die aus Manganoder Eisenoxydhydrat bestehen und wahrscheinlich bei der Umbildung des Dolomits ausgeschieden wurden. Auch die Entstehung des in den Gruben vorkommenden Braunsteins dürfte hierauf zurückzuführen sein.

Die Consistenz des Specksteins ist sehr wechselnd, von lockeren, zerfallenden Partien bis zu festen, schweren und zähen Slücken, die ein fast nephritarliges Aussehen haben. Beim Erhitzen wird er erst schwarz, brennt sich dann weiss und wird so hart, dass er Glas ritzt; hierbei verlicrt er zugleich die Eigenschaft, schwarz zu werden, was auf organische Substanz binweist, die jedenfalls auch bei seiner Bildung milwirkte. Das gleiche Verhalten zeigt indess auch der Dolomit, der davon den Namen "Schwarzkalk « erhalten hat.

Die Pseudomorphosen finden sich nun in den oben erwăhnten Knollen, 
rings in Speckstein eingewachsen, und bestehen selbst aus reinem Speckslein ohne eine Spur der ursprünglichen Substanz. Auch in den plattenförmigen Stücken, die Spaltausfüllungen darzustellen scheinen, finden sich namentlich Quarzkrystalle, die von beiden Seilen angeschossen und durcheinandergewachsen, aber ebenfalls vollständig pseudomorphosirt sind. Die Krystallform ist uberall mit der grössten Feinheit erhalten, und die Pseudomorphosen nach Quarz, die theils einzeln eingewachsen, theils zu Drusen vereinigt vorkommen, geben bekanntlich die feine Riefung der Prismen, die verschiedene Ausdehnung der Flächen, sowie alle Wachsthums- und Verwachsungserscheinungen, die den Quarz charakterisiren, mit einer Schönheit wieder, die künsllich kaum nachgeabmt werden könnte. Und dieselbe Feinheit in der Ausbildung zeigen die Bitterspathkrystalle, die namentlich sehr häufig durch sattelförmige Krummung und treppenförmige Verwachsung auf ihren Ursprung hinweisen.

Die Flächen dieser Pseudomorphosen sind glatt und spiegelnd und lassen sich im Dünnschliff, wenn sie von der Schliffebene durchschnilten werden, sehr leicht an parallel gelagerten und deshalb im polarisirten Lichte zugleich auslöschenden Schuppen aus der ubrigen wirr-filzigen Masse erkennen. Sehr häufig zeigen solche Schliffe durch reinen Speckstein ganze Systeme von parallel orientirten Streifen, welche der Spaltbarkeit des Dolomits nach $+\boldsymbol{R}$ entsprechen, die Stücke brechen dann mit grösster Leichtigkeit nach diesem Rhomboëder. Die Krystallflächen und die Spaltungsebenen nach $+R$ sind auch nicht selten durch einen Absatz von Eisenoxydhydrat ausgezeichnet, doch ist dies durchaus nicht, wie B l u m behauptet, stets der Fall, sondern es finden sich namentlich die Quarzpseudomorphosen häufig ohne jede Spur eines solchen Absatzes. Der Speckstein zeigt sich unter dem Mikroskop als einheitliche Masse. Nauck spricht zwar überall von einem Gehalt an Magnesiahydroxyd und Brucit, dies erklärt sich aber leicht daraus, dass er den Wassergehalt, den die Analyse giebt, fur Hydratwasser ansieht. Ueberdies müsste sich der Brucit in Salzsäure lösen, während diese thatsächlich nichts als einen Theil des Eisengehaltes wegfuhrt.

Ge in itz glauble in den Pseudomorphosen Quarzkörner zu erkennen, doch ist die Wahrscheinlichkeit viel grösser, dass diese in lebhaften Polarisationsfarben erscheineñden Theilchen, die erst bei sehr dünnen Schliffen zum Vorschein kommen, Talkschuppen sind, die parallel der Spaltbarkeit getroffen wurden, denn man muss stets berticksichtigen, dass man es mit einem schuppigen und nicht mit einem faserigen Aggregat zu thun hat. Ausserdem spricht noch der Umstand für diese Ansicht, dass sie ebenso häufig sich in den Pseudomorphosen nach Dolomit, sowie im dichten Speckstein finden.

Auch die chemische Zusammensetzung lässt nirgends derartige Bestand- 
theile vermuthen, sie stimml nach mehreren mit möglichst reinem, mikroskopisch geprüften Măterial ausgeführten Analysen mil der Formcl eines sauren melakieselsauren Magnesiasalzes $\mathrm{Mg}_{3}\left(\mathrm{SiO}_{3}\right)_{4} \mathrm{H}_{2}$ ziemlich genau uberein. Im Folgenden gebe ich eine Uebersicht über einige von mir ausgeführte Analysen, wobei jedoch ein geringer Thonerdegehall zum Eisenoxyd*) gezogen und das Wasser durch Verlust bei andauerndem, heftigem Glühen bestimmt ist.

I. ist die aus der Formel berechnete Zusammensetzung;

II. ist aus einer gelblichweissen Pseudomorphose nach Quarz,

III. aus einer rein weissen Dolomilpseudomorphose,

IV. aus einem gewöhnlichen grunlichen Speckstein.

I.

\begin{tabular}{lc}
$\mathrm{SiO}_{2}$ & 63,52 \\
$\mathrm{MgO}$ & 31,72 \\
$\mathrm{Fe}_{2} \mathrm{O}_{3}$ & - \\
$\mathrm{H}_{2} \mathrm{O}$ & $\mathbf{4 , 7 6}$ \\
\cline { 2 - 2 } & $\mathbf{1 0 0 , 0 0}$
\end{tabular}

II. 62,87 31,62 1,31 3,93
III. 63,32 31,49 0,57 $\begin{array}{r}4,38 \\ \hline 99,76\end{array}$
IV. 62,98 31,36 1,85 4,32

Die Abweichung von der Formel ist demnach so gering, dass mañ sie leicht auf Rechnung der analytischen Ungenauigkeiten selzen kann.

Da nun sowohl die mikroskopische Untersuchung, als auch die chemische Analyse uns deutlich darauf hinweisen, dass wir es bei diesen Specksteinpseudomorphosen nur mil dem letzten Endresultat eines langwierigen Processes zu thun haben, und uns der Speckstein selbst nicht die geringsten Mitlel in die Hand giebt, den Weg zu verfolgen, den dieser Process eingeschlagen hat, so sind wir genöthigt nachzuforschen, ob nicht vielleicht doch Reste des ursprünglichen Minerals, des Quarzes, oder Uebergänge zu erkennen sind. Und in der That findet sich neben dem Speckstein sehr bäufig frischer Quarz, der auch auf Drusen auskrystallisirt ist.

Er ist meist radialstrablig ausgebildet und uber Speckslein abgelagert, der eine raube, nierig-kugelige Oberfläche bat. Auf diese passen dann die Quarzkappen und ahmen sie so genau nach, dass man bei makroskopischer Betrachtung den Uebergang vor sich zu haben glaubt. Unter dem Mik roskop jedoch ist dieser Quarz klar und ohne Sprünge, nur manchmal entbält er Specksteinpartikel eingeschlossen, welche aber als abgerundete Partien ohne Verbindung daliegen, so dass man sie für mechanische Einschlüsse halten muss. Der Quarz selbst zeigt im polarisirten Lichte keine einbeitliche Auslöschung, vielmehr kommt eine eisblumenähnliche Parquettirung zum Vor-

*) Es ist indess sehr fraglicb, ob alles Eisen als Oxyd vorhanden ist, oder ob nicht vielmehr ein Theil als 0xydul einen geringen Theil der Magnesia isomorph vertritt, da, wie schon oben angeführt, nicht der ganze Eisengehalt durch Salzsäure ausgezogen wird. 
schein, die in allen Stellungen in den schönsten Farben erstrahlt. Es ist dieses die gleiche Erscheinung, die man an manchem Chalcedon beobachten kann, und jedenfalls eine Folge der radialstrahligen Textur. Nicht selten sind diese Quarze zellig angefressen und zeigen Eindrücke von später wieder aufgelösten Galcilkrystallen; auch wirkliche Zellenquarze finden sich in den Gruben.

Man durfte es bei diesen Quarzen mit Bildungen zu thun haben, die späterer Entstehung sind als der Speckstein und jedenfalls nicht gleichaltrig mit jenen, welche das Material zu den Pseudomorphosen geliefert haben. Denn wenn auch häufig Spuren einer Zersetzung an ihnen zu benerken sind, so tritt dieselbe doch nur ganz äusserlich auf und macht sich namentlich in den Krystalldrusen bemerkbar. Die Krystalle zeigen sich dann an den Spitzen cavernös und zerfressen, cariösen Zähnen nicht unähnlich. In den Grubchen und Höhlungen finden sich Reste von Speckstein, während der an exponirlen Stellen gebildete einfach von den Lösungen weggewaschen und forlgeführt wurde. Die Krystalle zeigen sich im Innern klar, und die Zersetzung ist nur ganz oberflächlich. Man kann dies leicht mit einem später eingetretenen, weniger intensiven Zersetzungsprocess erklären, der vielleicht bis in unsere Zeit fortdauert. Die Strahlen stehen uberall senkrecht auf den eingeschlossenen Specksteinpartien und an der Grenze zwischen Quarz und Speckstein zeigt sich nirgends auch nur die geringste Uebergangszone, frischer Quarz und reiner Speckstein schneiden scharf an einander ab.

Allerdings macht dann die Erklärung Schwierigkeit, wovon der Speckstein seine kugelig-nierige Oberfläche erhalten hat. Doch kommen uns hierbei andere Slücke zu Hülfe, welche ganz aus reinem Speckstein bestehen, aber Höhlungen zeigen, deren Oberfläche ebenso nierig-kugelig ist, und ähnlich ist auch das Innere der seltener vorkommenden, hohlen Quarzpseudomorphosen beschaffen. Man hat hieruber ebenso, wie uber die oben besprochenen beerenförmigen Absätze von Speckstein uber Bitterspath, die verschiedensten Theorien aufgestellt. Die Einen glaubten sie als Pseudomorphosen nach Magnesit oder Brucit auffassen zu müssen, die sich wohl anderwärts in ähnlichen Gestalten finden, aber in den Gruben und deren Umgebung gar nicht vorkommen; Andere erklärten sie für umgewandelten Chalcedon, wobei sie namentlich auf das Vorkommen in den hohlen Quar"zpseudomorphosen hinwiesen und damit die Theorie der Umwandlung des Quarzes in amorphe Kieselsäure zu beweisen glaubten. Mir scheint es viel wahrscheinlicher, dass man es hier mit einem primären Absatz von Speckstein zu thun hat, der sich wohl auch, wie andere feinkrystallinische Aggregate, da wo seine Masse zur Ausfullung des gebotenen Raumes nicht hinreicht, in traubig-nierigen Formen absetzt. Die beerenförmigen Absätze jedenfalls wurden direct als Speckstein gebildet, sei es dass dieser selbst in Lösung 
war oder dass er sich beim Zusammentreffen verschiedener Lösungen bildete. Der letzle Process durfte die meiste Wabrscheinlichkeit für sich haben, da eine Auflösung von Speckstein in den Gruben nicht beobachtet ist. Wohl aber gelang es mir, durch sechsstündiges Kochen von reinem Speckstein unit concentrirter Kalilauge $6,53 \%$ seines Gewichtes zu lösen.

Einen weiteren Beweis für das Vorkommen von jüngeren Quarzbildungen glaube ich in einem Stucke zu erblicken, welches zwei Quarzkrystalle ein Stuck Speckstein einklemmend zeigt, das an verschiedenen Stellen herausragt und unregelmässig eckig ist. Auch hier ist der Quarz klar.

Ausserdem sind die bekannten prachtvollen Morione hierher zu zählen, die in den Gruben in prächtigen, frischen Exemplaren gefunden werden; ebenso die, indess ziemlich seltenen, Absätze von Amethyst. Ebenso sicher späteren Ursprungs sind Quarzkrystalle, welche uber nierigem Speckstein liegen und an den Kanten und Ecken Eindrucke von diesem zeigen. Am allersichersten jedoch wird ein späterer Absatz von Quarz durch kleine, wasserhelle Bergkryställchen bewiesen, welche sich auf einem völlig pseudomorphosirten Quarzkrystall angesetzt haben*).

Endlich finden sich noch breccienartige Gemenge von Speckstein und Quarz, in denen der Quarz das Bindemittel lieferte.

Aber nicht allein als Quarz findet sich die freie Kieselsäure im Specksteinlager; so giebt es hier Feuersteine, die ebenso wie die der Kreide eine weisse Rinde haben, und namentlich sind Bildungen von Ghalcedon und Hornstein, oft in nächster Gesellschaft von Quarz, sehr häufig. Auch amorphe Kieselsäure findet sich; sie bildet hauptsächlich dünne Ueberzüge uber Quarzkrystallen, und diese Erscheinung ist es, die fruher für den Beginn der Pseudomorphosirung gehalten wurde. Dass diese Auffassung indess nicht zutrifft, folgt daraus, dass diese Ueberzüge nicht nur nabezu die Härte des Quarzes besitzen, sondern sich auch leicht durch Druck absprengen lassen, wobei unter ihnen die glatte Krystallfäche des Quarzes zum Vorschein kommt. Auch chemisch liess sich in diesen Ueberzugen keine Magnesia nachweisen und im polarisirten Lichte erscheinen sie völlig amorph. Aehnliche Bildungen finden sich auch als Ueberkleidung von Specksteinknollen und zeigen dann deutlich die traubig-nierige Oberfläche derselben. Auch uber Bitterspath-Krystallen und -Pseudomorphosen finden sich derartige Absätze, und es ist hier jedenfalls. am Platze, einer eigenthümlichen Pseudomorphose zu erwähnen, die im Zusammenhange damit vorkommt, nämlich von Chalcedon nach Bitterspath.

*) Das einzige Belegslück hierfür befindet sich in der Sammlung des Herrn Dr. S chmidt in Wunsiedel, der mir mit grosser Freundlichkeit das Studium seiner umfassenden Sammlung geslattete, wolür ich ihm hier nochmals meinen besten Dank ausspreche. 
Es findet sich an deujenigen Stücken, an welchen die Billerspalbpseudomorphosen einen Ueberzug von Kieselsüure tragen, nicht übcrall unter demselben Speckstein, sondern ein Theil der Rhomboëder besleht gleichfalls aus Kieselsäure, welche amorph zu sein scheint, mit wenigen krystallinischen Ausscheidungen, die rundlich und radialstrahlig sind, so dass im parallel polarisirten Lichte das schwarze Kreuz zum Vorschein komml; man wird das Mineral deshalb am besten als Chalcedon bezeichnen.

Sie zeigen die Spaltbarkeit des Bitterspathes, und zwar meist durch Absatz von Eisenhydroxyd auf den Spaltrissen, an. Es scheint, als ob dabei keine Zufuhr von Magnesia stattgefunden hätte, sondern die vorhandene Magnesia zur Umbildung eines Theiles in Speckstein verwendet worden wirre, während ausgeschiedene Kieselsäure den freien Platz einnahm; es geht nämlich uberall dieser pseudomorphe Ghalcedon in Speckstein über, von dem er auch Partikel eingeschlossen enthält.

Sowohl die Ueberzlige von amorpher Kieselsüure, als auch der in derben Massen auftretende Ghalcedon und Hornstein kommen in Speckstein pseudomorphosirt vor. Manchmal ist die amorphe Substanz trube und brüchig geworden und enthält Specksteinschüppchen. Am deutlichsten wird diese Umwandlung jedoch durch einen ganz in Speckstein pseudomorphosirten Quarzkrystall, der aus einem rein weissen Knollen herausbrach. Es sprang nämlich von demselben beim Ansetzen der Präparirnadel eine ganz dünne Schicht ab und darunter zeigte sich eine glänzende Fläche des umgewandelten Quarzkrystalles: Verhälnnisse, welche die mit einer dünnen Schicht amorpher Kieselsäure uberzogenen frischen Quarzkrystalle genau ebenso zeigen.

Ein merkwürdiges Vorkommen möchte ich hier noch erwähnen, das früher ziemlich häufig, jetzt aber kaum mehr gefunden wird, nämlich die Pseudomorphose yon Speckstein nach Kalkspath. Dieselben finden sich in Drusen zusammengehäuft; die Krystallform zeigt das charakteristische Kalkspathskalenoëder $R 3$ und auch die sonstige Beschaffenheit unterscheidet sich von allen anderen in den Gruben vorkommenden Pseudomorphosen. Denn während diese glalte Flächen besitzen und jede Unregelmässigkeit der äusseren Form des ursprünglichen Minerals genau nachahmen, zeigen diese Kalkspathformen eine rauhe, drusige Oberfläche, welche an die Pseudomorphosen von Bitterspath nach Kalkspath erinnert. Ich glaube daher annehmen zu darfen, dass wir es hier mit einer doppelten Umwandlungs-Pseudomorphose zu thun haben, indem der ursprungliche Kalkspath sich zu Bitterspath umgestaltele, dieser aber hinwiederum der Umwandlung in Speckstein unterlag. Bei dieser letzteren wurde dann die For'm der ersten Pseudomorphose genau erhalten und sogar die Spaltbarkeit des Bitterspaths ist deutlich in den Skalenoëdern zu erkennen.

Im Obigen habe ich die Vorkommnisse beschrieben, die sich in der 
Tiefe der Gruben selbst finden. Sie geben uns, so bestechend ihr Aeusseres für das blosse Auge sein mag, bei der mikroskopischen Untersuchung keine Anhalıspunkte für die Entslehung der Pseudomorphosen, da sie sich durch die Art ihres Vorkommens als spälere Bildungen zu erkennen geben und in gar keinem Zusammenhange mit dem fertigen Speckstein stehen.

Im Hangenden des eigentlichen Specksteins jedoch, der sich erst in einer Tiefe von circa $40 \mathrm{~m}$ von Tage aus findet, ist ein mulmig zerselzter krystallinischer Schiefer anstehend, in welchem häufig grössere Quarzaggregale ausgeschieden sind. Diese zeigen meist ganz ähnliche Formen, wie die secundären Quarze der Gruben, d. h. sie sind radialstrahlig ausgebildet, wobei oft an den einzelnen Strahlen Krystallfächen hervortreten, oder aber man hat es mit scheinbar ganz derben Quarzen zu thun. Bei genauerer Betrachtung entdeckt man auch in letzteren einzelne glänzende Krystallflächen, die sich aus der derben Grundmasse herauslösen. Häufig finden sich auch hier Krystalldrusen. Sowohl die radialstrahligen und die auskrystallisirten Quarze, als auch die derben Massen sind selten frisch, sondern sehen meist trube, verwaschen und zersetzt aus.

Diese Stucke nun ergaben bei einer eingehenden mikroskopischen Untersuchung genügende Anhaltspunkte für Schlüsse über die Entstehung der Specksteinpseudomorphosen.

Die derben Quarze, welche beim Zerschlagen oft schon dem blossen Auge erkennbare Krystallflächen zeigen, haben unter dem Mikroskop eine typisch porphyrartige Structur, indem grössere oder kleinere Quarzkrystalle in eine offenbar jungere Quarzgrundmasse eingebettet sind. Sie erscheinen kaum je frisch, meist sind sie weisslich trubbe und haben einen feltartigen Glanz ; sie sind weicher und spröder als der ursprüngliche Quarz und splittern beim Rilzen. Unter dem Mikroskop erscheint dann in den eingewachsenen Krystallen eine zonale Trübung parallel dem Prisma, seltener parallel dem Rhomboëder; dieselbe löst sich bei stärkerer Vergrösserung in ein System von feinen Capillaren auf; daneben sieht man auf den Sprungen, welche theils unregelmässig die ganze Masse durchsetzen, theils parallel dem Rhomboëder gehen, feine Specksteinschuppchen abgeselzt, die sich namentlich auch an die eingewachsenen Kryställchen anlegen (Taf. VII, Fig. 2). In einzelnen Partien, in denen sich die Sprünge mebr häufen, ist auch die Menge des gebildeten Specksteins grösser, und vorzugsweise planzt sich die Umbildung in die eingewachsenen Krystalle fort; dann erscheinen auch auf den zonal im Innern der Krystalle angeordneten Canälen die feinen Schuppen (Taf. VII, Fig. 3), bis in gewissen Stadien der ganze Krystall in Speckstein umgewandelt erscheint, wahrend die umgebende Grundmasse nocb aus weniger zersetztem Quarz besteht. Die Specksteinschuppen häufen sich bei weilerer Entwickelung des Processes auch in der Grundmasse immer mehr, bis endlich nur noch eckige Quarzkörner in 
einer filzigen Specksleinmasse liegen (Taf. VII, Fig. 4); diese Körner sind meist noch frisch, seltener durch Canälchen getrübt, und es lassen sich die einzelnen ursprünglichen Individuen leicht im polarisirten Lichte an der verschiedenen Orientirung der lebhaft gefärbten Partien erkennen. Eine Grenze von eingewachsenen, umgewandelten Quarzkrystallen, die sich durch parallele Auslöschung der Specksteinschuppen charakterisirt hätle, konnte ich in diesen Stücken nicht feststellen.

Dass die Umwandlung zuerst die Krystalle ergreift, bevor die Grundmasse derselben unterliegt, ist um so bemerkenswerther, als sonst die Krystalle furr viel unangreifbarer gelten, als die derben Massen. Dieser Process lässt sich indess auch makroskopisch verfolgen, indem man manchmal aus solchen Stucken Krystalle herausschlagen kann, welche oberflächlich mit einer dünnen, glänzenden Specksteinschicht überzogen sind, die alle Eigenschaften der Oberfläche der Pseudomorphosen zeigt. Entfernt man dieselbe, so erscheint darunter der Krystall angefressen und geätzt. Auch fertige Specksteinpseudomorphosen in einer Quarzgrundmasse sind mit blossem Auge zu erkennen.

Seltener geht der Process der Pseudomorphosirung auf scheinbar anderem Wege vor sich, indem sich der Speckstèin auf einzelnen, besonders geeigneten Punkten absetzt und daselbst häuft. Bei genauerer Untersuchung findet man jedoch, dass die einzelnen Specksteinpartien sich an Stellen abgesetzt haben, wo der Quarz von einem sehr engmaschigen Netze von Sprüngen durchzogen ist, so dass die winzigen Quarzkörner sehr bald in dem sich bildenden Speckstein aufgehen. Diese Verhältnisse zeigt Fig. 5, Taf. VII. Bei weiter fortschreitender Zersetzung wird das Bild, welches diese Quarze im Dünnschliff darbieten, gerade das entgegengesetzle von dem in Fig. 4 abgebildeten; während dort Quarzkörner in eine Specksteinmasse eingebettet lagen, finden wir hier Specksteinpartikel in einem Netzwerke von Quarz, aber dieselben sind uberall durch feine Risse unter einander verbunden (Fig. 6). Das Endresultat unterscheidet sich natürlich bei beiden Arten der Zersetzung nicht.

Ausser in diesen derben, typisch porphyrartig ausgebildeten Massen findet sich der Quarz auch in radialstrahligen Aggregaten, welche gleichfalls hin und wieder Krystalle eingebettet enthalten, die dann beim Zerschlagen herausspringen. Unter dem Mikroskope zeigen diese Quarze ganz dieselben Verhältnisse wie die derben, nur dass der Specksteinabsalz meist von den Ansatzstellen der Strahlen ausgeht. Es finden sich dann nicht selten in den sehr splitterigen Quarzen zwischen den Strahlen grössere Specksteinpartien, oder die ganzen Strahlen sind von feinen Schuppen umgeben. Dass sich dieses strahlige Gelüge nach der Pseudomorphosirung nicht erhalten hat, ist leicht verständlich, wenn man bedenkt, dass die Grenzen der Strahlen weder krystallographisch bestimmte, noch ebene 
Flächen waren, also viel weniger orientirend auf die Specksteinschuppen einwirkten, als die echten Krystallfläichen ${ }^{*}$ ).

Selten sind endlich feinkörnige Quarze, welche natürlich um so mehr der Zersetzung unterliegen, je feiner das Korn ist, da sich damit auch die Zahl der Wege, welche die zersetzenden Lösungen in das Innere führen, vermehrt.

Alle im Vorhergehenden beschriebenen, im Mulm über dem eigentlichen Specksteinlager vorkommenden Quarzvarietäten sind auch auf Drusen auskrystallisirt und lassen hier ebenfalls Anzeichen der Zersetzung erkennen, welche auf sehr verschiedene Weise vor sich gegangen ist. Die einen sind oberflächlich abgewaschen, Kanten und Flächen sind verschwunden und die Krystalle ragen nur noch als Spitzen hervor. Hier durfte man eine einfache Auflösung der Quarzkrystalle mit Recht annehmen. Andere zeigen sich ebenso cavernös von oben her zerfressen, wie die später gebildeten Quarze des Specksteinlagers. Wieder andere zeigen eine äusserlich glänzende, aber vielfach zerrissene Krystallfläche. Diese Risse nabmen sich die den Speckstein erzeugenden Lösungen als Weg und zersetzten das Innere der äusserlich noch frischen Krystalle. Einige endlich zeigen nur eine innere Trubung, die man beim Abbrechen des Krystalles als mit dem Prisma parallele getrubte Zonen erkennt; auch sie besteht, wie die Trubung der eingewachsenen Krystalle, aus feinen Canälchen.

Auch auf diesen Krystallen haben sich, wenn auch ziemlich selten, secundäre Quarze abgesetzt; so findet sich ein Hohlraum in einem stark zersetzten, feinkörnigen Quarz mit kleinen wasserbellen Quarzkryställehen ausgekleidet, wobei die Grenze des ursprünglichen und des späteren Minerals deutlich zu erkennen ist.

Im Vorhergehenden habe ich die feinen Schuppchen und Aggregate, die sich auf den Sprüngen der Quarze absetzten, der Kürze halber einfach als Speckstein bezeichnet, obgleich es mir nicht gelang, direct eine chemische Analyse derselben anzustellen, da sie wegen ihrer Feinheit nicht von der umgebenden Quarzmasse zu trennen waren. Dass es indess unzweifelhaft Speckstein ist, erhellt schon aus der ununterbrochenen Reihe von Uebergängen, die zum Schlusse zu echtem Speckstein und zwar ausschliesslich zu Speckstein führen. Andererseits sind die optischen Verhältnisse genau die des Specksteins und die mit dem gemengten Material angestellten Analysen ergaben, neben einem Eisengehalt, stets Magnesia. So enthielt ein splitterig gewordener strahliger Quarz 3,26\% $\mathrm{MgO}$, während

*) Es befindet sich jedoch ein Stück radialstrahligen Specksteins in der hiesigen Slaatssammlung, das eine auffallende Aehnlichkeit mit den radialstrahligen Quarzen besilzt. Dasselbe stammt indess nichl von Göpfersgrün, sondern aus dem Pullenreulher Bergwerke, das dem anderen Kalkzuge angehört. 
in einem der feltglänzenden, derben sogar $5,62 \% M g O$ gefunden wurden.

Ueberall werden diese Specksteinpartikel direct von Quarz begrenzl und das Bild, das die Zwischenstufen bei diesem Processe bieten, hat, natürlich abgesehen von der eigenthümlichen Structurform, die grösste Aehnlichkeit mit einem sich bildenden Serpentin. Schon hierdurch wird es wahrscheinlich gemacht, dass wir es mit einer Umwandlungspseudomorphose zu thun haben. Jedenfalls ging aber dieser Process direct vor sich, und namentlich findet sich nie und nirgends amorphe Kieselsäure als Zwischenproduct zwischen Quarz und Speckstein.

Wo wir uberhaupt amorphe Kieselsäure im Specksteinlager finden, ist nicht der geringste Anhalt vorhanden, dass an ihrer Stelle vorher Quarz gewesen ist. Im Gegentheil, sie findet sich selbständig neben dem Quarz und unterliegt für sich der gleichen Umwandlung. Möglich ist ja immerhin, dass ein Theil derselben aus den Quarzen ihren Ursprung herleitet, indem auch bei einer Umwandlungspseudomorphose ein Theil der Quarzsubstanz weggeführt werden musste, was auch die feinen Canälchen, die doch am wahrscheinlichsten durch Auslaugung entstanden sind, zur Genige zu beweisen scheinen. Es löste sich dieser Theil des Quarzes in den kohlensauren Alkalien*), die die Gewässer führten und schlug sich aus denselben amorph nieder.

Was bei der makroskopischen Betrachtung die meisten Schwierigkeiten machte, war der Umstand, dass sich die Pseudomorphosen in einer ganz gleichartigen Specksteinmasse eingebeltet finden; denn freistehend wurden dieselben noch nie gefunden, was auch leicht erklärlich ist, da die Weichheit und geringe Consistenz des entstehenden Specksteins eine Erhaltung der Form im freien Raume uberhaupt nicht gestattet hätte. Es kann indess diese porphyrartige Ausbildung nach der mikroskopischen Betrachtung nicht die geringste Schwierigkeit bereiten, indem schon der Quarz vor seiner Umbildung; wie wir sahen, die gleiche Structurform darbietet. Für einige Stücke, namentlich solche, an denen die Pseudomorphosen nach Quarz und Bitterspath zugleich auftreten, dürften indess auch die Stücke vom Strehlerberg bei Redwitz eine genugende Erklärung abgeben, in welchen Quar'zkrystalle innig mit Bitterspathkrystallen verwachsen sind. Man braucht also nur in den seltensten Fällen eine Ausfüllung des hohlen Raumes durch den so schwer beweglichen Speckstein anzunehmen, und dies ist am wahrscheinlichsten da der Fall, wo unter einer nierig-kugeligen Oberfläche

*) Quarz ist durchaus nicht so unlöslich, als man gewöhnlich annimmt; so lösten sich von einem reinen, feinpulverisirten Bergkrystall bei sechsstündigem Kochen in concentrirler Kalilauge 27,5\%, während sich von einem trüben Göpfersgrüner Vorkommen $39,46 \%$ gelöst hatten. 
Pseudomorphosen nach Quarz und Bitterspath zum Vorschein kommen. Hier ist eine gewisse Wahrscheinlichkeit vorhanden, dass sich über den Krystalldrusen vor der Umwandlung Speckstein abgeselzt hat und so die Erhaltung der Form ermöglichte. Auch diese Art des Processes wird durch die beerenförmigen Absätze von Speckstein ưber Bitterspath in der Natur angedeutet.

Wollte man indessen eine blosse Ausfüllungspseudomorphose annehmen, so stehen schon einige Stücke bei makroskopischer Betrachtung dieser Ansicht entgegen. Wenn man nämlich in den plattenförmig ausgebildeten Stücken die von beiden Seiten angeschossenen Quarzkrystalle betrachtet und sieht, wie diese durcheinander gewachsen, und wie alle gegenseitigen Eindrücke erhalten sind, so muss man sich von der Unhaltbarkeit dieser Theorie uberzeugen. Auch eine Verdrängung von Molekul zu Molekul dürfte nicht viel Wahrscheinlichkeit für sich haben, da die Specksteinsubstanz sehr schwer beweglich ist, und die Wegführung der Kieselsäure des Quarzes und ihre Ersetzung durch das kieselsaure Magnesiasalz, den Speckstein, sich äbnlich anbören würde, wie wenn man eine Pseudomorphose von Pyromorphit nach Bleiglanz für Verdrängung erklären wollte. Man könnte ja auch hier mit eben solchem Recht den Pyromorphit von Lösungen als solchen herfubren lassen.

Es bleibt demnach nur noch die Wahrscheinlichkeit einer directen Umwandlung von Quarz in Speckstein. Eine Entscheidung über die ganze Frage kann aber nur auf dem Wege des Versuchs gefunden werden, d. h. durch den experimentellen Nachweis, dass der krystallisirte Quarz im Stande ist, direct aus Lösungen von Magnesiasalzen die Base aufzunehmen und mit ihr ein Silicat zu bilden, das in der Zusammensetzung und in den physikalischen Eigenschaften dem Speckstein entspricht. Da uns aber naturlich im Laboratorium nicht jene unendlichen Reihen von Jahren zu Gebote stehen, mit denen die Natur arbeiten kann, so mussen wir diese durch die Intensität unserer Mittel erselzen und diese sind : hohe Temperatur, starker Druck und Concentration der Lösung.

Es wurde zunächst ein Gemenge von reinem, mikroskopisch gepruftem Quarz, der aus wasserklarem Bergkrystall durch Zerkleinern gewonnen war, mit einer wässerigen Lösung von Kaliumcarbonat und Magnesiumsulfat acht Tage lang gekocht; um das Gemenge in permanenter Bewegung zu erhalten, wurde vermittelst eines Gasometers ein ununterbrochener, schwacher Luftstrom durch einen umgekehrten Trichter in die Lösung eingeleilet. Nach acht Tagen wurde der ungelöste Theil abfiltrirt, mit Königswasser ausgekocht und geglüht. Der Quarz zeigte sich nun trübe, gerade wie der naturliche im ersten Stadium der Zersetzung, und uberall waren dazwischen schuppige Aggregate abgelagert. Das Gemenge ergab bei einer chemischen Untersuchung 1,1\% $\mathrm{MgO}$. 
Hierauf wurde die oben beschriebene Mischung in einem luftdicht verschlossenen Elintenlaufe acht Stunden lang auf etwa $320^{\circ}$ erhitzt. Die hierbei gewonnene Flüssigkeit wurde wie oben weiter behandelt und der gereinigte und gegluhte Rückstand einer weiteren Untersuchung unterworfen. Das Resultat war ein ähnliches, nur war die Menge der schuppigen Aggregate zwischen den Quarzkölnern grösser und die Analyse ergab 2,86\% $M g O$. Um nun zu erfahren, ob die Specksteinbildung leichter durch amorphe Kieselsäure zu Stande komme, wurde solche möglichst rein dargestellt und unter den gleichen Bedingungen ebenso lange auf $320^{\circ} \mathrm{er}-$ hitzt. Auch hier zeigten sich die gleichen Erscheinungen, nur war ein Theil der vorher völlig amorphen Kieselsäure in eine Chalcedon-ähnliche Masse umgewandelt, welche Aggregatpolarisation zeigte; die chemische Analyse ergab nur 2,6\% $\mathrm{MgO}$, was jedenfalls beweist, dass die amorphe Kieselsäure durchaus nicht in höherem Grade zur Specksteinbildung geeignet ist, als der krystallisirte Quarz.

Wurde nun neben Quarz und schwefelsaurer Magnesia weniger Kaliumcarbonat angewandt, so zeigte sich eine Verminderung der Einwirkung, bis sich beim gänzlichen Fehlen des Kaliumcarbonates die Magnesiaaufnahme des Quarzes nur eben noch nachweisen liess. Eine ähnliche Reihe von Resultaten ergab die Anwendung von Magnesiumcarbonat an Stelle des Sulfats.

Auch bei anhaltendem Kochen von Magnesiumcarbonat mit einer verdünnten, alkalischen Wasserglaslösung wurde Speckstein hervorgebracht.

In allen diesen Versuchen zeigt sich, dass Kieselsäure und Măgnesia, wenn sie unter den verschiedensten Umständen in Lösungen zusammenkommen, stets ein und dasselbe Silicat bilden, das in seinem optischen Verhalten durchaus dem Speckstein gleicht.

Was nun die Frage betrifft, woher die Magnesia zur Umwandlung des Quarzes und Kieselsäure und Magnesia zur Umwandlung des Dolomits es lässt sich doch kaum annehmen, dass der Dolomit in der Nähe des Granits schon vor seiner Umwandlung zu Speckstein so viel Quarz enthalten habe, um bei dieser Umwandlung jede Kieselsäurezufuhr entbehren zu können - gekommen sind, so haben die Mineralogen schon frühe mit Recht auf den zerstörten und zersetzten Granitstock von Thiersheim hingewiesen. Wenn auch der Granit selbst wenig Magnesia enthält, so finden sich doch Umwandlungen, die viel Magnesia erfordern, nicht selten in der Umgebung von Graniten. Ich erinnere hierbei nur an den Pseudophil von Chipal in den Vogesen, der ebenfalls an der Grenze des Granits aus Magnesia-freiem Feldspath entstanden ist.

Wenn man sich nun fragt, in welcher Form die Magnesia dabei aufgetreten ist, so muss man zunächst die Theorie von Blum, welcher gasförmige Magnesiaexhalationen annimmt, obne weiteres zurückweisen, 
da sie allen chemischen Gesetzen direct widerspricht. Ich möchte mich für heisse Wässer aussprechen, welche die Magnesia, sei es als kohlensaures oder als schwefelsaures Salz enthielten und die Eruplionen der Granile ibegleiteten. Spricht ja doch der ganze Habitus der Granite weniger für einen feurigen, als für einen sogenannten wässerigen Schmelzfluss. Und auch sonst bieten nicht selten Schichten von Kalk oder ähnlichen Gesteinen, die sich in der Nähe derartiger Eruptivgesteine finden, ein Bild dar, das sich am ungezwungensten mit einer Durchtränkung mittelst Lösungen, die bald Kieselsäure, bald Magnesia und andere Stoffe enthielten, erklären lässt; ich erinnere hierbei nur an die schönen Contactwirkungen, die im Fassathal so typisch ausgebildet sind. Ob man indess mit $\mathrm{Nauck}$ einen Wechsel in der Durchtränkung des ursprünglichen Kalkstein- oder Dolomitlagers annehmen darf, scheint mir zweifelhaft, denn nirgends ist z. B. nachzuweisen; dass der Dolomit zuerst Magnesit geworden wäre, ebe er zu Speckstein metamorphosirt wurde; im Gegentheil, die Specksteinschuppen sind direct aus dem echten Dolomit gebildet und liegen in diesen eingebettet. Ob man nun eine wechselnde Durchwässerung des durch die Eruptionen gelockerten Lagers, oder eine gleichzeitige Durchtrảnkung mittelst verschiedener Lösungen annehmen soll, scheint mir immerhin von geringer Bedeutung; jedenfalls wird es beim eingehenden Studium des Göpfersgrüner Vorkommens zur evidenten Gewissheit, dass der Speckstein nicht als solcher von den Lösungen zugeführt wurde, was ja auch durch die schon oben besprochene schwere Löslichkeit der Specksteinsubstanz unwahrscheinlich gemacht wird.

Ist nun durch die oben beschriebenen Versuche eine Umwandlung von Quarz in Speckstein als möglich nachgewiesen, so mag es doch auffällig erscheinen, dass wir Pseudomorphosen von Speckstein nach Quarz sonst so selten finden. Zur Erklärung dieser Thatsache muss zunächst noch darauf aufmerksam gemacht werden, dass man freistehende Pseudomorphosen auch von Göpfersgrün nicht kennt, und dass die Zahl derjenigen, die sich unter einer Bedeckung von nierigem Speckstein gebildet haben könnten, wenn sie uberhaupt sicher nachgewiesen werden, eine sehr geringe ist; die freistehenden wurden, wegen der geringen Widerstandsfähigkeit des Materials, das sich bei der Umwandlung bildete, von den dabei thätigen Lösungen selbst direct zerstört und weggeführt, so dass die Form nicht erhalten bleiben konnte und wir nur den ersten Beginn dieser Pseudomorphosirung zu beobachten vermögen. Ebensowenig lässt sich bei einer Umwandlung von derbem Quarz zu Speckstein irgend ein Anhaltspunkt für die sichere Feststellung des ursprunglichen Minerals auffinden. Wenn man nun bedenkt, wie selten derartige, porphyrisch ausgebildete Quarze sind, wo die Krystalle von Quarz später durch Lösungen von einer derben Grundmasse umgeben wurden, wenn man das Zusammentreffen dieses Umstandes 
mit der günsligen Nähe des Granits in Betracht zieht, durch dessen Eruption das ganze Lager eine Störung erlitten hat und, in seinem Gefüge erschuttert, den circulirenden Gewässern zạhllose Wege öffnete, wenn man endlich die tiefgehende Zersetzung dieses Granites selbst betrachtet, so kann man sich der Ueberzeugung nicht verschliessen, dass nur die Häufung all' dieser hervorragend günstigen Bedingungen ein so grossartiges Resultat haben musste, wie es uns in dem Speckstein zu Göpfersgrün vorliegt.

Es werden indess noch mehrere ähnliche Vorkommen erwähnt, die jedoch, so lange sie chemisch nicht analysirt sind, mit grosser Vorsicht aufgefasst werden durften, da sich schon manches, mit diesen Pseudomorphosen identificirte Stuck als eine ganz verschiedene Substanz herausgestellt hat. Ich führe im Folgenden die wichtigsten dieser Vorkommnisse an.

Fow le ${ }^{*}$ ) erwähnt Pseudomorphosen von Speckstein nach Quarz aus dem körnigen Kalke von Newton in New Jersey und nach Brogniart**) sollen sich ähnliche im Thale Viège am Mte. Rosa finden. Blum spricht von einem Vorkommen von Reichenbach im Odenwalde, wo bei Versuchsbauen auf Erze Quarzkrystalle mit einer Rinde von Speckstein gefunden worden sein sollen. Ebenfalls in Verbindung mit Erzen steht ein Vorkommen vom Romanus Erb-Stollen bei Freiberg $\left.{ }^{* * *}\right)$.

Dagegen werden die von Emmons f) im Serpentin von Middlefield in Massachusets entdeckten und "Hampshirite " genannten Pseudomorphosen nicht hierher zu rechnen sein, da ein Wassergehalt von $15 \%$ dagegen spricht. Die Vorkommnisse von Zinnwald, Altenberg ff) in Sachsen, Ehrenfriedersdorf gehören ebenfalls kaum hierher, sondern eher zum Kaolin. Sicherer dagegen sind die Funde im Gyps bei Stecklenburg im Harz $+\dagger \dagger$ ) und die von Bu rkhart in der Grube Concepcion zu Bolanos*t) in Mexico beobachteten Knollen hierher zu rechnen, doch ist in beiden Fällen der Quarz derb, so dass der Uebergang weit schwieriger zu verfolgen ist. Es durfte daher auch sonst mancher Speckstein, dessen Ursprung weder durch die Form noch durch Uebergänge nachgewiesen werden kann, aus Quarz entstanden sein. So z. B. das von $\mathrm{G} u \mathrm{mb}$ el bei Hof im Fichtelgebirge beobachtete Vorkommen.

Jedenfalls glaube ich nachgewiesen zu baben, dass einer Umwandlung von Quarz in Speckstein durchaus keine chemischen Hindernisse im Wege

*) Sill im an's American Journal 1832, 320.

**) Brogniart, Traité élémentaire de Minéralogie 1807, 1, 497.

***) Frenzel, Mineralogisches Lexikon für Sachsen, 304.

t) Hitch cock, Report on the Geology etc. of Massachusets 1837, 368.

$+t)$ Magazin für die Oryktografie Sachsens. Heft V, 189.

$+t+$ ) Zeitschrift der deulschen geologischen Gesellschaft $2,136$.

*.t) Burkhart, Aufenthalt und Reisen in Mexico 139. 
stehen; dass im Gegentheil der Speckstein dasjenige Mineral ist, das sich beim Zusammentreffen von Quarz und Kieselsäure uberhaupt mit Magnesiahaltigen Lösungen am leichtesten bildet; dass aber nur die besonders gunstigen Bedingungen, die bei Göpfersgrün vorliegen, diese Zersetzung deutlich verfolgen lassen und es bewirkten, dass sich auch im fertig gebildeten Speckstein die Form so genau erhalten hat.

Vorstehende Arbeit wurde auf Anregung des Herrn Prof. Groth im Laboratorium der mineralogischen Staatssammlung zu München ausgefuhrt, und befindet sich in letzlerer eine vollständige Collection von Belegstucken zu den im Vorhergebenden beschriebenen Vorkommnissen des Specksteinlagers von Göpfersgrün. 